\title{
Diffraction Contrast Scanning Transmission Electron Microscopy - A Bend- contour-free Way to Image Dislocations, Voids and Precipitates
}

\author{
Yuanyuan Zhu', Colin Ophus², Mychailo B. Toloczko' and Danny J. Edwards' \\ 1. Energy and Environmental Directorate, Nuclear Sciences Division, Pacific Northwest National \\ Laboratory, Richland, WA 99352, USA \\ National Center for Electron Microscopy, Molecular Foundry, Lawrence Berkeley National \\ Laboratory, Berkeley, California 94720, USA
}

Studying radiation effects on the microstructural and mechanical properties of structural materials used for nuclear reactor core components is critical for clean and safe implementation of nuclear energy. Neutron irradiation, or any high-energy ion irradiation, can displace atoms, leading to the continual production of cascades of defects. Over time these defects can evolve into larger features such as dislocation lines and loops, voids and bubbles, and various types of precipitates that all affect bulk mechanical properties. To understand how these irradiation-induced defects govern the changes in materials behavior, it is essential to characterize these defects individually as well as collectively to understand how they form and evolve during irradiation.

Ever since the early development of transmission electron microscopy, dislocation analysis has been in the purview of conventional TEM (CTEM), supported by well-developed diffraction contrast theories for understanding the contrast of defects in CTEM micrographs. However, diffraction contrast imaging (DCI) technique in the scanning transmission electron microscopy (STEM) mode has been proposed as early as 1970's as an alternative to CTEM, offering additional advantages including alleviated bend contours and allowing observations in thicker foil. Recently, Phillips et al demonstrated that the dynamical theory of diffraction contrast, including the $\boldsymbol{g} \boldsymbol{\bullet} \boldsymbol{b}$ criteria for defect imaging in CTEM, can be valid in STEM mode[1]. Yet, it is not clear why DCI STEM suppresses the bend contour nor how different STEM parameters affect the contrast of dislocation images. To address these questions, we investigated the image formation process in the STEM mode and its reciprocal relationship with the CTEM [2]. As shown in Figure 1, the bend contours in the bright field (BF) STEM image were effectively negated when the STEM collection semi-angle was increased to $3 \mathrm{mrad}$. Unlike the parallel electron beam in CTEM, the converged STEM electron probe opens up a cone in reciprocal space that accesses the rocking-curve oscillation in the Kossel-Möllenstedt fringes; then a sufficiently large $\boldsymbol{\beta}_{\mathrm{s}}(\sim$ $\boldsymbol{\alpha}_{\text {s) }}$ behaves like a "mask" averaging out this oscillation, leading to a uniform background when imaging dislocations. It is particularly powerful in resolving complex microstructures containing a high density of dislocations, grain boundaries, voids and precipitates.

Using a HT-9 ferritic/martensitic alloy as an example, we further discuss practical guidelines regarding STEM parameters and specimen orientation and thickness are provided for DCI STEM imaging. As the DCI STEM imaging technique has been established and optimized, it is beneficial to take advantage of the associated analytical techniques that can be performed in tandem with imaging to provide a comprehensive microstructural and chemical characterization. A workflow of such an attempt is schematically illustrated in Figure 2, with an emphasis on three main types of irradiation defects dislocation (lines and loops), voids and precipitates. This streamlined characterization can facilitate the comparison among nuclear alloys with different irradiation history and conditions. 
References:

[1] PJ Phillips, MC Brandes, MJ Mills and M De Graef, Ultramicroscopy, 111 (2011) 1483-1487.

[2] Y Zhu, C Ophus, MB Toloczko and DJ Edwards, submitted.

[3] The authors acknowledge funding from the U.S. Department of Energy Office of Nuclear Energy's Nuclear Energy Enabling Technologies program project CFA 16-10570, Office of Fusion Energy Sciences under contract DE-AC05-76RL01830, and for Molecular Foundry which is supported by the Office of Basic Energy Sciences, under Contract No. DE-AC02-05CH11231.

TEM-BF mode

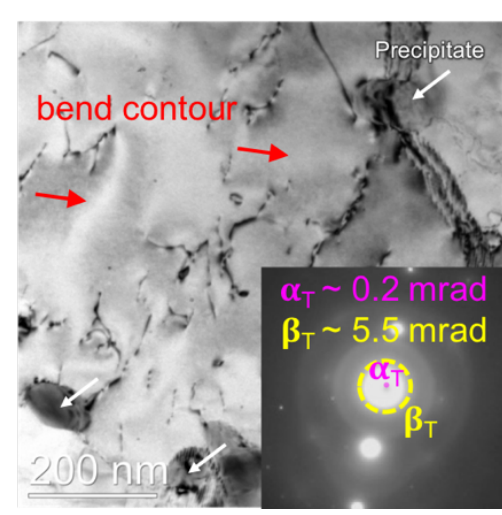

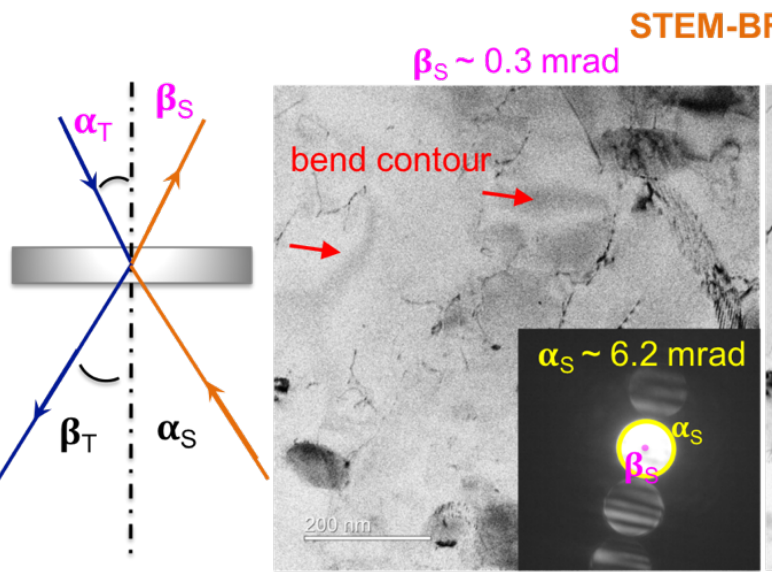

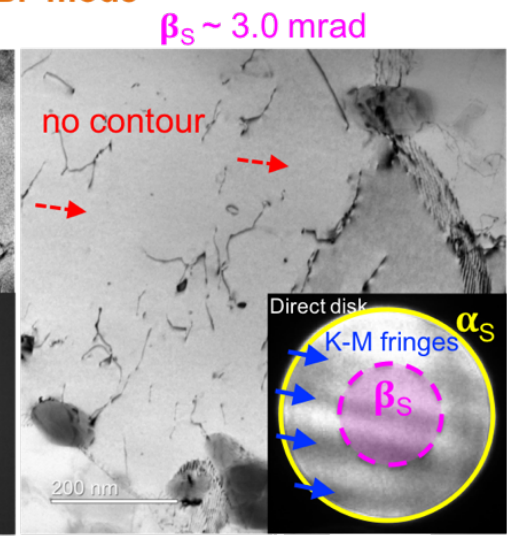

Figure 1. DCI STEM dislocation imaging is advantageous over the CTEM at suppressing bend contour by averaging out the rocking-curve oscillation in reciprocal space.

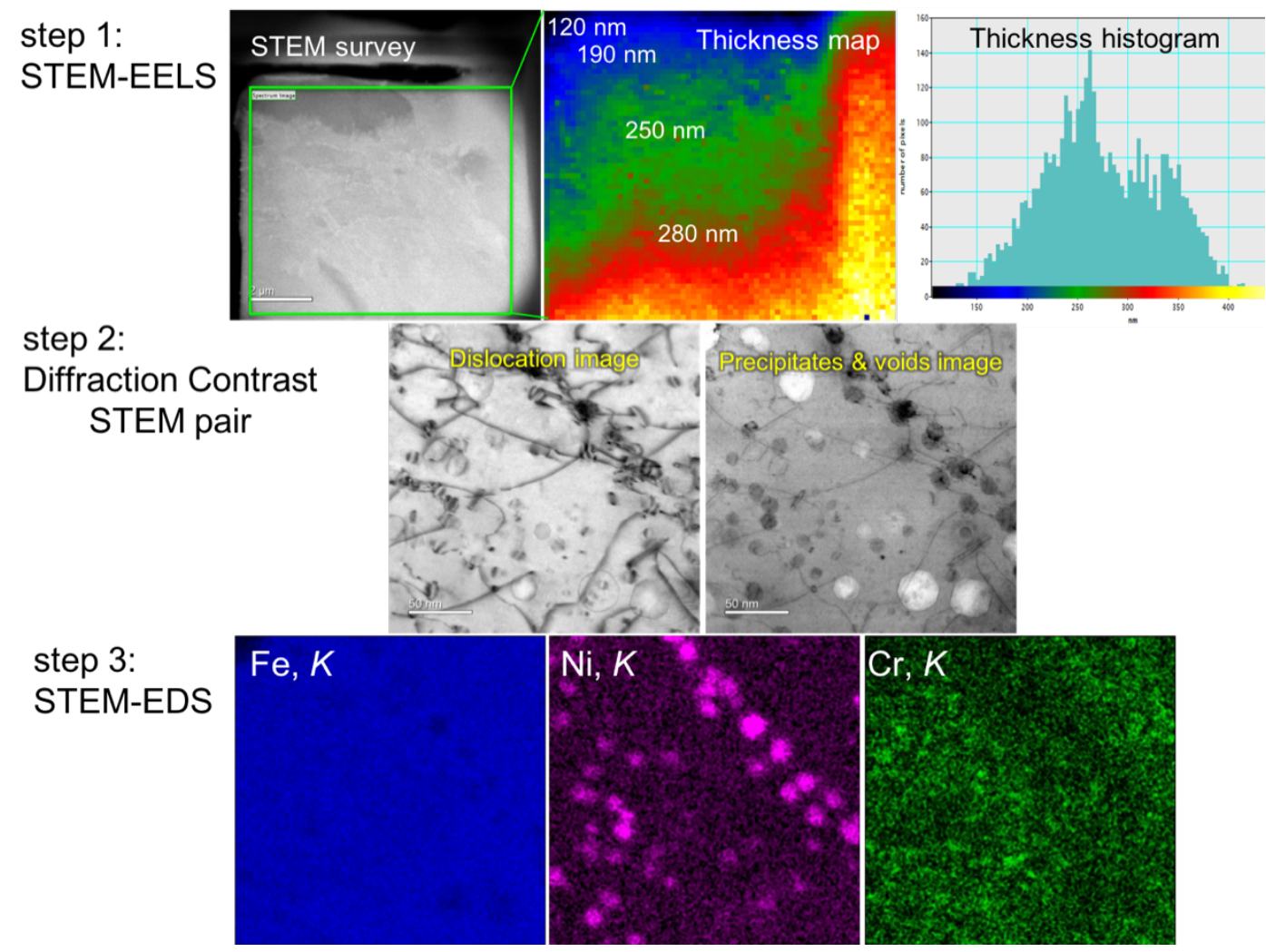

Figure 2. The workflow of a comprehensive defect characterization in STEM mode for a BCC Ferric nuclear reactor structural alloy HT-9. 